\title{
Ciencia CreActiva. ¿Cómo sensibilizar a docentes y estudiantes de ámbitos educativos no universitarios en la economía circular?
}

\author{
CreActiva Science Project. How to Raise the Awareness of Non- \\ University Teachers and Students Regarding the Circular Economy?
}

\section{Raquel Fernández-Cézar', Felipe Gértrudix-Barrio'1, Manuel Gértrudix-Barrio ${ }^{2}$ y Natalia Solano Pinto ${ }^{1}$}

${ }^{1}$ Universidad de Castilla-La Mancha

${ }^{2}$ Universidad Rey Juan Carlos

\section{Resumen}

\begin{abstract}
Este artículo forma parte de un proyecto de investigación financiado por la FECYT (Espańa) que busca una mejora educativa en el ámbito de la sostenibilidad y la tecnología relacionada con la economía circular entre el alumnado preuniversitario de la Comunidad de Castilla-La Mancha (Espańa). La educación es la más adecuada vía de entrada de la conciencia ciudadana respecto de la economía circular, y las acciones en los docentes permean después a su alumnado. Por ello, en este artículo se midió el grado de sensibilización de los docentes de educación no universitaria en relación con la economía circular, se determinó el tipo de acción didáctica que podía ser efectiva y se presenta una intervención educativa integral llevada a cabo con ellos a través de un curso de formación semipresencial. El principal recurso didáctico utilizado fue el video, con entrevistas a mujeres científicas y a expertos educativos en ámbitos de sostenibilidad y economía circular. La metodología empleada fue cuantitativa en el análisis de la sensibilización del profesorado (para lo que se ha empleado como instrumento un cuestionario), y cualitativa para el análisis del tipo de acción didáctica (grupo focal de expertos). Los resultados acerca del nivel de sensibilización del profesorado obtenidos arrojan que el interés de los docentes por la economía circular es medio-bajo, y que entre los interesados el grado de compromiso y preocupación se sitúa entre alto y muy alto (83\%). Las dimensiones analizadas en el grupo focal apuntan a la adecuación curricular, las medidas educativas, las metodologías, los recursos y materiales. Al término de la intervención presentada se espera obtener un repositorio de videos creados por los propios estudiantes que puedan ser integrados en nuevas propuestas educativas, favoreciendo, con ello, un mayor compromiso entre la comunidad educativa ante esta problemática.
\end{abstract}

Palabras clave: cuestionario, economía circular, educación no universitaria, sensibilización, formación del profesorado, video didáctico.

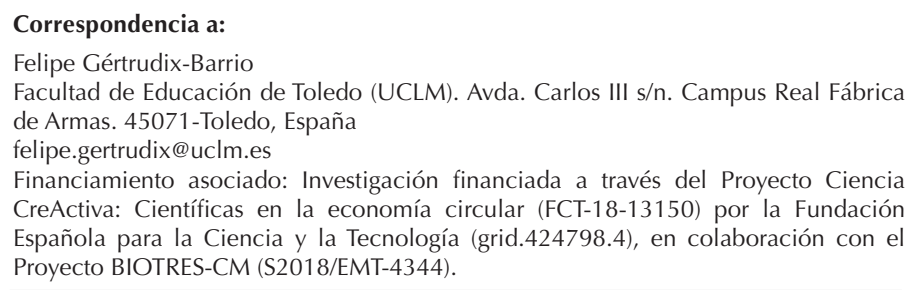

(c) 2020 PEL, http://www.pensamientoeducativo.org - http://www.pel.cl

ISSN:0719-0409 DDI:203.262, Santiago, Chile doi: 10.7764/PEL.57.2.2020.4 


\section{Abstract}

This article is part of a research project funded by the FECYT (Spain) that seeks to improve education in the field of sustainability and technology related to the circular economy among pre-university students in the Castilla-La Mancha community in Spain. Education is the most appropriate entry point for citizen awareness of the circular economy, and actions by teachers subsequently permeate their students. Therefore, this article measures the degree of awareness of non-university education teachers regarding the circular economy, determining the type of didactic action that may be effective, and presents a comprehensive educational intervention carried out with them through a blended learning course. The main teaching resource used was video, with interviews with women scientists and educational experts in the areas of sustainability and the circular economy. The methodology used was quantitative for the analysis of teacher awareness, with a questionnaire being used as an instrument, and qualitative for the analysis of the type of educational action, for which a focus group of experts was used. The results obtained regarding the level of teacher awareness show that the interest of teachers in the circular economy is medium-low and that among the stakeholders the degree of commitment and concern is between high and very high (83\%). The dimensions analyzed in the focus group included curricular adaptation, educational measures, methodologies, and resources and materials. At the end of the intervention described, the expectation was to obtain a repository of videos created by the students themselves that could be integrated into new educational proposals, thus promoting greater commitment among the educational community to this problem.

Keywords: awareness, circular economy, didactic video, non-university education, questionnaire, teacher training.

\section{Introducción}

Cuando hablamos de residuos sólidos urbanos (RSU) denominados residuos domésticos (Ley 22/2011, de 28 de julio, de Residuos y Suelos Contaminados) nos referimos a todos aquellos restos que se generan debido a la acción de la actividad doméstica y comercial en las ciudades y pueblos. Los efectos causados por este tipo de residuos son múltiples como la contaminación atmosférica, edáfica o de suelo y de las aguas subterráneas, entre otros.

En 2007 la cantidad total de residuos generados mundialmente fue de 3,2 Gigatoneladas (Gt), de las que 1 Gt fue reciclada o reusada, 0,7 Gt fue incinerada, convertida en gas, en fertilizante o áridos, y 1,5 Gt fueron depositadas en vertederos (Tisserant et al., 2017). Este flujo de materiales contiene enormes cantidades de recursos potencialmente utilizables, y supone una gran oportunidad para fomentar el desarrollo de la economía circular (en adelante EC), donde los Estados tienen un papel importante y decisorio, ya que son los que deben esgrimir las medidas necesarias y avivar el fuego del cambio (Inocêncio da Silva \& Ferreira Maia, 2017). Sin embargo, la realidad es que el porcentaje de reciclaje o reutilización de los RSU sigue siendo muy escaso. En España, por ejemplo, supone solo un 43,3\% (Seguí, Medina y Guerrero, 2018), situándose lejos de lo que la directiva europea se comprometió en 2015: aumentar el reciclaje de RSU hasta el 65\% y disminuir los RSU que van a vertederos hasta el 10\% en 2030. Para ello, sería necesario aumentar el reciclado de 97 Megatoneladas (Mt) en los países de la Unión Europea. 
La problemática climática se ha convertido en una amenaza global que pone en riesgo la sostenibilidad del modelo de producción vigente, el cual puede llevar a un futuro donde los recursos fundamentales como el agua dulce se conviertan en motivo de conflicto por su escasez. Tanto es así, que se estima que la extracción de recursos de la Tierra es 1,7 veces mayor que la capacidad sostenible (Soto, 2018). Además, el extractivismo es un problema local que facilita una solución global, constituyendo el primer eslabón en las cadenas internacionales de comercialización y producción (Gudynas, 2015; 2018).

Por su parte, el mal reciclaje de ciertos productos que contienen materiales no biodegradables produce no solo un coste medioambiental, sino también económico, pues se estima que el plástico que acaba en los mares y océanos afecta negativamente a sectores como el del turismo o la pesca, con pérdidas de hasta 13 billones de dólares (Kaplan, 2015).

Ahora bien, para alcanzar estas premisas debemos actuar sobre la base de la formación ciudadana, esto es, sensibilizar a los más pequeños y a sus formadores en el conocimiento y el reconocimiento a través de la apropiación del concepto. Esto hace indispensable que existan estrategias de educación ambiental efectivas para desarrollar un sentido de cuidado y respeto por el entorno, tanto en las generaciones presentes como en las futuras (Comisión Europea, 2014; European Comission, 2017). El desenmascaramiento y valoración de esta problemática se torna esencial, como afirma Molano (2012), ya que combatir el conformismo cultural es el primer paso para conseguir un cambio paradigmático.

Desde esta perspectiva, una educación ambiental idónea es aquella en la que los sujetos individuales que conforman una sociedad consiguen adquirir una conciencia colectiva por la que apreciar la conservación del ecosistema, de tal manera que caminen hacia un uso propicio y efectivo de la materia prima, cuyo beneficio será así más longevo (Macedo y Salgado, 2007). Los objetivos de este tipo de educación, además, pasan por conocer la problemática y las directrices a seguir, pues las posibles consecuencias de la implementación del trabajo a nivel curricular representan distintos aspectos relacionados con inquietudes sociales y ambientales (Maya, 1998).

Si estas medidas se hicieran efectivas, la educación ambiental sería la piedra angular de las programaciones en todos los contextos, teniendo en cuenta las particularidades de cada área en específico (Torres Consuegra, 1996). Algunos autores como Monclus y Sabán (1999) ya establecieron tres alternativas en este sentido: establecer los temas transversales como contenidos que planteen un posicionamiento — tanto individual como colectivofrente a las dificultades a mbientales; relacionar los contenidos con valores y actitudes que permitan al alumnado desarrollar de manera autónoma reflexiones mediante las cuales favorecer conductas personales; y los contenidos deben incluirse en las áreas curriculares ya establecidas.

Teniendo en cuenta las premisas antes expuestas, el presente estudio tuvo como objetivo prioritario buscar una mejora educativa en el ámbito de la sostenibilidad, así como la tecnología relacionada con la EC y la reutilización de los RSU entre los alumnos de nivel preuniversitario, para el desarrollo de una intervención educativa. Para ello, se ha acometido una actuación sobre los dos ejes fundamentales de la EC: profesorado y alumnado.

\section{De una economía lineal a una EC}

Desde que en 1972 se produjera la primera conferencia sobre el clima en Estocolmo (Organización de las Naciones Unidas, ONU, 1973) — más conocida como la Primera Cumbre de la Tierra-, y a pesar de los conflictos generados por los intereses económicos entre los gobiernos de los distintos Estados, surgió una preocupación internacional por el medio ambiente vinculada con cuestiones técnicas, demográficas y culturales (Boudes, 2011). Esta primera "legislación blanda" relativa a cuestiones internacionales relacionadas con el medio ambiente constituyó el inicio de ciertos cambios hacia nuevos modelos de actuación más "verdes". Así, a partir de un crecimiento 
económico y social inaceptables, los participantes acordaron principios y recomendaciones para la acción en el plano internacional, porque se comprendió que un trabajo coordinado entre la dimensión socioeconómica y la ambiental permitiría que el desarrollo sostenible y la conciencia medioambiental se relacionaran de manera eficaz, siempre y cuando se privilegiaran las estructuras integradas internacionalmente como:

- la planificación y ordenación de los asentamientos humanos desde el punto de vista de la calidad del medio (Recomendaciones 1 a 18);

- la ordenación de los recursos naturales y sus relaciones con el medio (Recomendaciones 19 a 69);

- agentes contaminantes a nivel internacional (Recomendaciones 70 a 94);

- los aspectos educacionales, informativos, sociales y culturales relacionados con el medio ambiente (Recomendaciones 95 a 101);

- el desarrollo del medio (Recomendaciones 102 a 109).

Todas estas recomendaciones señalaron "los límites de la racionalidad económica y los desafíos que genera la degradación ambiental al proyecto civilizatorio de la modernidad” (Leff, s.f., cuarto párrafo).

De igual modo, este espacio otorgó la materia prima para el desarrollo de una visión intelectual, moral, social y espiritual acerca del medio ambiente, porque se entendió la necesidad de que los hábitos de consumo debían cambiar para evitar que la destrucción del ecosistema llegara a ser irreversible (Ramírez y Sánchez y García, 2004).

Todas estas ideas están implícitas en el concepto de EC, que ya fue defendido por Pearce y Turner (1990) quienes demostraron el impacto que la economía de la época tenía sobre el medio ambiente, analizando el modelo de economía lineal y sus efectos. En la misma dirección, Stahel y Reday (1981) entendieron la economía como un sistema circular con materiales constantemente fluyendo, lo cual permitía cuidar al máximo el gasto, facilitaba la creación de nuevos empleos y la mejora de la eficiencia al innovar en nuevas tecnologías. Entendiendo los alcances de estas miradas, la Comunidad Europea fue una de las precursoras a nivel mundial en adoptar este tipo de medidas, creando la primera Directriz sobre Protección del Medio Ambiente y los Consumidores, y el primer Programa de Acción Ambiental (1973) cuyo resultado fue el Libro Verde como táctica para moderar los residuos provenientes de combustibles fósiles (Comisión Europea, 2013).

Como concepto, podemos decir que la EC es aquella economía capaz de aprovechar la durabilidad de los productos, que promueve el ecoconsumo, la reutilización y la reparación, el reciclado de residuos, el mercado de las materias primas secundarias, así como los "nuevos modelos de negocio como la ecología industrial, la simbiosis territorial, la economía de la funcionalidad, la economía colaborativa, etc.” (Economía Circular, 2017, p. 5).

Se opone, por tanto, a la actual economía lineal de extraer, fabricar, consumir y tirar, puesto que los tres principios de la EC son: conservar y mejorar el capital natural; optimizar los rendimientos de los recursos; y fomentar la efectividad del sistema. Lo anterior solo es viable si se regenera, se comparte, se optimiza, se crea, se virtualiza, y se realiza un uso apropiado de los residuos para permitir un impulso económico que apueste por la productividad de los recursos, promoviendo el capital natural y su resiliencia (Webster, 2017). Un término claro hasta para un nińo (Fernández, 2018), pero que no se practica habitualmente, ya que para ello se debe tomar una postura regenerativa y ecocéntrica que actúe sobre aspectos concretos que impidan ese cambio de mentalidad (Sandoval-Ruiz y Ruiz-Díaz, 2019), por ejemplo, la mejora de la calidad de los productos y la eficiencia en el proceso de venta y reciclaje (Stahel \& Reday, 1981) y, en general, seguir una serie de preceptos fundamentales como: el diseño de la prevención de residuos, la construcción de resiliencia a través de la diversidad, el uso de energías renovables, los residuos son comida, el pensamiento en sistemas, el pensamiento en cascadas y el enfoque en el rendimiento (Fundación COTEC para la Innovación, 2019). 
No obstante, armonizar las acciones humanas y el crecimiento económico con la protección del medio ambiente no es un reto fácil, ya que se deben romper los paradigmas dominantes que más perjudican, de modo que la racionalidad ambiental pasa irremediablemente por convertirnos en seres humanos integrados con la naturaleza (Leff, 2004).

Desde esta óptica, un aumento en la concienciación social para alternar el modelo económico presente actuará de forma directa en un uso adecuado de los residuos urbanos y, con ello, en la generación de nuevos productos de alto valor añadido por parte de los agentes sociales (ciudadanos, instituciones, etc.).

\section{Sensibilizar, educar y construir en EC y RSU}

Las experiencias educativas en torno al reciclaje de plástico, vidrio, madera o cartón han sido profusamente desarrolladas por diversas organizaciones (Apud Porras, 2012; Ecoembes.es, s/f), y universidades (Wadel, Pérez, Giacomelli, Scheffer y Pascual, 2018).

Por el contrario, los RSU hasta la fecha han tenido un menor impacto mediático y, por ende, existen menos acciones de sensibilización ciudadana. Esta situación contrasta con la necesidad imperativa de alcanzar un cambio en la forma de tratamiento de este tipo de residuos, como queda reflejado en toda la normativa que se ha desarrollado en el ámbito de nuestra investigación, y a la que se le está dando forma definitiva en las leyes europeas, nacionales y regionales (Centro para el Desarrollo Tecnológico Industrial, CDTI, 2016; España Circular 2030, 2018; Gobierno de España y Comisión Europea, CE, 2017; Ley de Economía Circular de Castilla-La Mancha, 2019).

Por otra parte, es evidente que una buena estrategia de EC pasa ineludiblemente por realizar grandes esfuerzos en el ámbito de la información, sensibilización y educación, y que estos además estén dirigidos a todo tipo de público (Economía Circular, 2017). Por tanto, se hace necesario el desarrollo de proyectos piloto (Fernández Cézar, Pinto-Solano y Muñiz-Hernández, 2018), a través de acciones educativas en centros de formación inicial (primaria y secundaria), en los que se desplieguen tareas de sensibilización, como por ejemplo, para disminuir el consumo (haciendo conciencia acerca del coste ecológico de elaborar un producto) o para aprovechar el reciclaje de residuos, dándole un nuevo valor ańadido, lo cual permitiría visualizar las enormes posibilidades que tiene la bioeconomía en el lugar donde se vive (Buckland, 2007). Esto se puede observar en el caso de Castilla-La Mancha, que al ser una comunidad eminentemente de producción agrícola "genera una importante contribución a la emisión de gases de efecto invernadero, que se ve incrementada con el desperdicio y generación de residuos, así como la pérdida de materia orgánica para el suelo" (Avilés Pozo, 2018).

\section{La EC y la educación}

Como ya hemos dicho, el término EC se opone a la economía lineal. Por ello, una de las propuestas de la directiva europea es incentivar económicamente a los fabricantes de productos verdes, para apoyar la recuperación y el reciclaje. Pese al esfuerzo, la posibilidad de mejorar procesos y orientarlos hacia la EC recae sobre el sistema educativo y así formar personas con las competencias necesarias para conocer los procesos y los recursos relacionados con la EC, y que cursarán estudios en ramas de ciencias, tecnología, ingeniería, arte o matemáticas (STEAM, en inglés, por science, technology, engineering, arts and math).

Hasta lo que sabemos, no existen programas educativos preuniversitarios en España que contemplen el estudio de la EC. Así mismo, en el sistema educativo español a nivel universitario (grado, máster y doctorado) la oferta formativa varía desde solo un curso hasta un máster completo y en ellos se hace un tratamiento muy global del tema, sin acercar el análisis a las potencialidades concretas de las regiones (Ruiz-Pastor, Mulet, y Chulvi, 2018). Se 
reconoce, entonces, la necesidad de aumentar la presencia de la EC en estudios de STEAM y en el sistema educativo de las etapas inferiores, así como también de incrementar la oferta de formación en las distintas regiones en cuanto a los aspectos que implica la EC en cada zona y las posibilidades de empleo verde que conlleva su implementación.

Creemos que es importante el desarrollo de programas o acciones formativas STEAM que contemplen la EC a nivel preuniversitario, pues el conocimiento temprano favorece su interiorización, y puede tener incidencia en la elección de los estudios posteriores. Por otro lado, consideramos también fundamental que estas acciones se lleven a cabo de manera contrastada en entornos rurales y urbanos, pues en estos contextos las relaciones sociales son muy distintas (Salvia, Andreopoulou, \& Quaranta, 2018), así como también es conocido que el alumnado adolescente, debido al momento vital, presenta percepciones más estereotipadas que otras poblaciones (Barth, Kim, Eno, \& Guadagno, 2018; Stadler, Baganz, Vermeulen \& Keesman, 2015), mismas que se ven alimentadas por las concepciones que los maestros de los centros escolares rurales tienen acerca de la ciencia, según se reporta en el estudio de Ruiz Medina, Parga Lozano, y Martínez Pérez (2009). Sin embargo, igualmente con estos estudiantes estamos a tiempo de provocar un cambio en su percepción, la que aún no tienen formada y que es más susceptible de ser modificada que la de los adultos.

\section{Instrumentos para medir la percepción o sensibilización}

A pesar de la poca literatura científica en torno a la sensibilización respecto de la EC, existen algunos cuestionarios de la percepción y valoración del medio ambiente. Así, encontramos varios instrumentos con propiedades psicométricas confirmadas como la Encuesta de ecología y medio ambiente del Ministerio de Medio Ambiente (2010) y la Escala del nuevo paradigma ecológico, que incluye 16 creencias acerca de la relación de la humanidad con el planeta y del impacto de sus actividades sobre el mismo (Dunlap, Van Liere, Mertig \& Jones 2002; Vozmediano Sanz y San Juan Guillén, 2005). A pesar de ello, en ninguno de estos estudios se realiza un análisis predictor en cuanto a la conducta de reciclaje en los sujetos estudiados, lo que sí ocurre en el Cuestionario sobre la conducta de reciclaje basada en la conducta planificada (Aguilar Luzón, García Martínez, Monteoliva Sánchez y Salinas Martínez de Lecea, 2006) donde se utiliza la medida de las variables del modelo del valor, las normas y las creencias (VNC) hacia el medio ambiente y la de la conducta planificada (TCP), aspecto importante para poder discernir lo que se piensa que se debe hacer, o bien, el discurso y las acciones que luego conscientemente se llevarían a cabo.

\section{Investigaciones previas acerca de EC y educación}

Existe poca literatura científica en la que se haya trabajado de forma eficiente la sensibilización en torno a la EC. Algunos estudios recientes y de los que nos hacemos eco los encontramos en las investigaciones que se han realizado en algunos países como China, Finlandia o Italia en torno a la forma de iniciar el camino hacia el desarrollo sostenible (Guo \& Zhang, 2014). La inferencia más importante — coincidente entre dichos estudios-, es que a pesar de tener potencial e infraestructuras suficientes para desarrollar una buena política ambiental, los esfuerzos pueden resultar no fructíferos si la base de este crecimiento no es una educación ambiental de calidad, que vaya desde la base hasta llegar al nivel global, de tal manera que se establezca como un valor transversal (Åhlberg, Aineslahti, Alppi, Houtsonen, \& Nuutinen, 2014; Chen \& Chen, 2019).

Otros estudios se han dirigido a la creación de materiales gamificados, como por ejemplo el juego de mesa In the Loop que permite el aprendizaje respecto de los conceptos principales de la EC y de la caducidad de los materiales (Anderberg \& Thisted, 2015; Whalen, Berlin, Ekberg, Barletta, \& Hammersberg, 2017). 


\section{Objetivos}

Dada la situación educativa descrita, en este estudio se plantearon los siguientes objetivos:

- Medir el nivel de sensibilización respecto de la EC de profesorado no universitario.

- Determinar el tipo de acción que sería necesaria en educación.

- Presentar una intervención didáctica para contribuir al conocimiento y la concienciación de dicho profesorado.

\section{Método}

La metodología empleada en esta investigación fue cuantitativa con muestreo no probabilístico en cuanto a la medición del nivel de sensibilización del profesorado no universitario, y cualitativa en la determinación del tipo de acción necesaria y el diseño de la intervención didáctica integral.

Para alcanzar el primer objetivo, se utilizó un cuestionario basado en el que desarrollaron Sánchez-Emeterio y Figueira (2019) al que se añadieron términos relacionados con la economía circular en el que consideramos la medida del interés del profesorado no universitario por la EC junto con el número de inscritos en un curso ofrecido por el Centro Regional de Profesorado (CRFP) de Castilla La Mancha, Espańa y el número de esos docentes que completó el cuestionario y sus respuestas. A partir de estos criterios selectivos, los inscritos en el curso llegaron a 50 docentes participantes y de ellos 32 rellenaron completamente el cuestionario, los que terminaron representando finalmente la muestra de este estudio.

De estos 32 profesores, 23 eran mujeres, siete hombres, y dos no indicaron su sexo. En cuanto a las etapas educativas en las que ejercían su profesión, cinco de ellos eran docentes de educación infantil, cinco trabajaban en educación primaria, y el resto se desempeñaba en educación secundaria; 20 de ellos impartían clases de ciencias, y dos lo hacían en ramas de economía (Figura 1).



Figura 1. Diagrama de flujo sobre el proceso de selección de la muestra. 
El cuestionario utilizado incluía preguntas acerca de la preocupación del docente por el medio ambiente y su conocimiento de algunos temas relacionados con la EC, por ejemplo: "En una escala de 1 a 5 en la que $1=$ NADA preocupadola por el medio ambiente y el 5 = muy preocupadola, ¿en qué casilla te colocarías a nivel general?”.

Los términos respecto de los cuales fueron consultados los docentes correspondieron a: EC, ecosistema, modelo lineal de producción-consumo, desarrollo sostenible, decrecimiento económico en producción y consumo, sobreexplotación, contaminación, ciclo del agua, ciclos de reciclaje, cambio climático, aplicaciones y programas de bajo consumo, gasificación, química sostenible, alfabetización ecosocial, reciclado de vidrio, edificio de consumo cero, sistemas alimentarios sostenibles, tecnología productiva de alimentos sostenible y electrocatalizador de hidrógeno.

Por su parte, para alcanzar los otros dos objetivos restantes se utilizó la técnica del grupo focal de expertos. Sus resultados, junto con los obtenidos en el cuestionario a modo de triangulación, sirvieron para realizar una intervención didáctica global de formación y divulgación con el propósito de sensibilizar a los docentes del estudio en los conceptos de EC y, por ende, que ellos sirvieran de transmisores de dichos conocimientos en sus aulas de referencia.

\section{Resultados}

\section{Análisis del cuestionario}

Respecto al interés y preocupación que demuestran los docentes frente a la temática trabajada, el 43,75\% se declaró muy preocupado por el tema, el 40,63\% señaló sentirse preocupado, y el 12,5\% manifestó estar algo preocupado, sin que ninguno de ellos indicara encontrarse nada o casi nada preocupado.

En cuanto a su conocimiento acerca de los conceptos involucrados en la EC, el profesorado declaró que el término más desconocido para ellos era el "electrocatalizador de hidrógeno", seguido de "química sostenible". Entre aquellos términos de los que mayoritariamente indicaron conocer un poco se destacan la "economía circular", el "modelo lineal de producción-consumo", el "decrecimiento económico en producción y consumo", las "aplicaciones y programas de bajo consumo", la "gasificación", la "alfabetización ecosocial”, el "edificio de consumo cero", los "sistemas alimentarios sostenibles" y la "tecnología productiva de alimentos sostenible". Por último, entre los términos que declararon conocer perfectamente se cuentan "ecosistema", "desarrollo sostenible", "ciclo del agua", "contaminación”, "sobreexplotación” y "ciclos de reciclaje”.

Por su parte, los docentes se definieron más conocedores de aquellos términos que son más generales, quizás porque ya forman parte del vocabulario de prácticamente toda la ciudadanía, al estar más presentes en las informaciones de prensa. Contrariamente a ello, se declararon muy poco conocedores de tecnologías particulares como el electrocatalizador de hidrógeno, y algo más de las tecnologías relacionadas con los alimentos y el reciclaje (Figura 2). 




Figura 2. Grado de conocimiento terminológico de los docentes participantes respeto de la EC.

\section{Análisis del grupo focal}

Todos los expertos participantes en el grupo focal (cinco) han sido docentes vinculados con universidades y trabajan en el ámbito de la educación en distintas áreas. Constituyen, por tanto, un grupo multidisciplinar con experiencia en temáticas relativas al medio ambiente.

A partir de este análisis realizado se obtuvo la tipología de las acciones que son necesarias a nivel educativo.

El análisis del grupo de expertos estuvo centrado en cuatro tópicos de discusión: adecuación de contenidos acerca del desarrollo sostenible y la EC; medidas educativas para concienciar y sensibilizar a los jóvenes en esta materia; metodologías educativas más efectivas para provocar una sensibilización en los estudiantes; y tipo de recursos digitales más eficaces para trabajar este tipo de temáticas en el aula.

En la siguiente tabla se pueden ver los puntos de acuerdo entre los expertos. 
Tabla 1

Análisis de los tópicos del grupo focal de expertos

\begin{tabular}{|c|c|c|}
\hline Tópico & Puntos de acuerdo & Divergencias \\
\hline $\begin{array}{l}1 \text { Adecuación } \\
\text { curricular }\end{array}$ & $\begin{array}{l}\text { - Integración curricular. } \\
\text { - Interdisciplinar. } \\
\text { - Globalizada. }\end{array}$ & $\begin{array}{l}\text { - Depende del docente si quiere } \\
\text { integrar el tema o no. }\end{array}$ \\
\hline $\begin{array}{l}2 \text { Medidas } \\
\text { educativas }\end{array}$ & $\begin{array}{l}\text { - Hay que llegar a los jóvenes, ya que existe } \\
\text { una dualidad entre ellos (concienciados y } \\
\text { no concienciados). } \\
\text { - Falta conexión entre las instituciones y } \\
\text { los entornos. } \\
\text { - Existe una mayor presencia de acciones } \\
\text { educativas en torno a la EC en la } \\
\text { educación no formal. Se debe potenciar } \\
\text { este tipo de acciones en el ámbito de la } \\
\text { educación formal. }\end{array}$ & $\begin{array}{l}\text { - Excesiva responsabilidad } \\
\text { sobre el individuo y no sobre las } \\
\text { colectividades (“eco-agobio”). } \\
\text { - La sociedad destruye el } \\
\text { pensamiento crítico de } \\
\text { los estudiantes ante esta } \\
\text { problemática. } \\
\text { - La sensibilización existe, pero } \\
\text { en áreas concretas (ciencias). } \\
\text { Hay que incentivar de forma } \\
\text { transversal. }\end{array}$ \\
\hline 3 Metodologías & $\begin{array}{l}\text { - Activas: Aprendizaje en servicios, } \\
\text { aprendizaje basado en problemas, } \\
\text { aprendizaje basado en juegos, resolución } \\
\text { de problemas. } \\
\text { - El juego como estrategia es fundamental } \\
\text { (videojuegos, gamificación). } \\
\text { - Cuentos, excursiones, talleres. }\end{array}$ & $\begin{array}{l}\text { - Aprendizaje situacional. } \\
\text { - Recursos educativos abiertos } \\
\text { (REAs). } \\
\text { - Enseñar desde la práctica para } \\
\text { llegar a la conceptualización } \\
\text { (aprender haciendo). }\end{array}$ \\
\hline $\begin{array}{l}4 \text { Recursos y } \\
\text { materiales }\end{array}$ & $\begin{array}{l}\text { - Videoconferencia. } \\
\text { - Cualquier recurso atendiendo al estilo de } \\
\text { aprendizaje. } \\
\text { - Videojuegos, redes sociales (Tik Tok, } \\
\text { Instagram). }\end{array}$ & - Realidad aumentada. \\
\hline
\end{tabular}

Fuente: Elaboración propia.

Aunque existen ciertas divergencias, el 100\% de los expertos abogó por una inclusión de los conceptos relativos a la EC en el aula de forma transversal e interdisciplinarmente, ya que visualizan que no se trata de una problemática que atañe a una materia o área curricular, sino que su dimensión educativa debe enfocarse de forma integral.

En cuanto a las medidas educativas por tomar, se releva la responsabilidad de la escuela ante este tipo de problemáticas (80\%). La información que le llega al estudiante a través de los medios (Internet) no siempre es positiva, existiendo una clara dualidad entre los estudiantes más sensibilizados y entre aquellos que no lo están. De igual modo, los entornos familiares muchas veces son reflejo de una sociedad poco concienciada y solidaria, por lo que finalmente será la escuela la que dará respuesta y soluciones a esa carencia, creando ciudadanos críticos y responsables.

Llama la atención el término "eco-agobio", que si bien no todos los expertos lo señalan como un problema (60\%), empieza a tomar cada vez más relevancia incluso como un posible trastorno al que la Asociación Psicológica de Estados Unidos definió como un miedo crónico a la destrucción medioambiental: "A chronic fear of environmental doom”. (Whitmore-Williams, Manning Visiting, Krygsman \& Speiser, 2017, p. 68). 
Así mismo, los expertos reconocen que las metodologías activas resultan más eficaces que otras estrategias didácticas. El 100\% de ellos apostó por los aprendizajes basados en problemas o por las clases invertidas, mientras un $80 \%$ fue partidario del uso de la gamificación. Por último, el $60 \%$ se interesó por los recursos educativos abiertos (REAs), así como por el aprendizaje situado. En este sentido, pese a que en las aulas se cuente con tecnología avanzada, existe aún cierto recelo entre los docentes para utilizar algunas técnicas más innovadoras.

Finalmente, en cuanto a los recursos, los expertos creen que estos, ligados con las estrategias metodológicas, se deben utilizar en función de los estilos de aprendizaje del estudiante y existe un consenso mayoritario en cuanto al uso de la videoconferencia como recurso para lograr este propósito.

\section{Intervención educativa}

A partir del objetivo principal de este estudio que era buscar una mejora educativa en el ámbito de la sostenibilidad, la tecnología relacionada con la EC y la reutilización de los RSU entre el alumnado preuniversitario, se establecieron los siguientes objetivos didácticos y de innovación para el desarrollo de la intervención educativa:

- Contribuir al conocimiento de científicas españolas en el ámbito de la EC.

- Conocer el grado de sensibilización y conocimiento del profesorado acerca de los elementos que integran la EC.

- Fomentar la conciencia proactiva del alumnado respecto de esta temática mediante actividades de gamificación (concurso).

- Promover entre los docentes el uso de materiales didácticos orientados a la concienciación de la EC.

En esta etapa de la intervención, el desarrollo del trabajo consideró tres acciones principales: creación de un entorno web y materiales audiovisuales; creación e implementación de un curso de formación docente; y concurso de videos acerca de la EC.

\section{Acción 1: Creación de un entorno welb y materiales audiovisuales ${ }^{1}$}

El material audiovisual creado para el proyecto consistió en 10 entrevistas a mujeres científicas dedicadas a la investigación relacionada con la EC. Cada encuentro fue realizado en un formato de video didáctico (Gértrudix, Rajas, Barrera, Bastida y Soto, 2017) y con una estructura fija: presentación; historias de vida respecto de cómo y por qué llegaron a ser investigadoras, sus motivaciones, sus líneas de investigación más importantes y cuáles de ellas estaban ligadas a la EC, enfatizando los elementos químicos que utilizan y que están implicados en ella; y visión personal acerca de la igualdad y paridad de la mujer en la investigación y la ciencia.

1. Todo el contenido de la intervención, junto con los recursos creados, se puede visualizar en el siguiente enlace: https://cienciacreactiva.bio3project.es/ 




Figura 3. Ejemplo de presentación de los videos de mujeres científicas entrevistadas.

El 30\% de las mujeres entrevistadas correspondió a docentes universitarias, de ellas un 10\% trabajaba en empresas y un $60 \%$ lo hacía como investigadora del Centro de Investigaciones Científicas, CSIC. Todas ellas tenían a su cargo proyectos de investigación y solo un 30\% de ellas eran directoras de los mismos. En cuanto a sus principales temas de investigación destacan los siguientes tópicos: recuperación de residuos en el hogar, desarrollo de software y apps, agua potable, alfabetización y concienciación ecosocial, reciclado del vidrio, diseño de edificios de bajo consumo energético, industria agroalimentaria, química del hidrógeno, comida preparada o gasificación de productos orgánicos.

\section{Acción 2: Creación e implementación de un curso de formación docente}

El diseño metodológico (blended learning o aprendizaje semipresencial) utilizado para el tratamiento del curso se enfocó en la idea del microlearning, esto es, "pequeñas unidades de contenido y un diseño de actividades de aprendizaje a corto plazo" (Santiago, 2015, primer párrafo). Así, la estructura de cada unidad o módulo (Figura 4) constaba de una breve introducción de documentos de lectura y una serie de actividades que ayudaban a la comprensión de los contenidos. 


\section{¿Cuál es la estructura del curso?}

\begin{tabular}{|c|c|c|c|}
\hline Módulo & Descripción & Modalidad & Fecha \\
\hline Módulo 1: Presentación del curso. & $\begin{array}{l}\text { Presentación presencial del curso. } \\
\text { ¿Qué es la economia circular y qué puede aportar a la sociedad? }\end{array}$ & $\begin{array}{l}\text { Presencial } \\
\text { En las sedes de } \\
\text { los centros de } \\
\text { formación }\end{array}$ & $\begin{array}{l}\text { Tercera } \\
\text { semana } \\
\text { de febrero }\end{array}$ \\
\hline $\begin{array}{l}\text { Módulo 2. Mujeres científicas en la } \\
\text { economía circular. }\end{array}$ & $\begin{array}{l}\text { Videos didácticos sobre el trabajo de mujeres cientificas en ámbltos de sostenibilidad y/o o } \\
\text { economia circular. }\end{array}$ & On-line & Marzo \\
\hline $\begin{array}{l}\text { Módulo 3. La voz de los expertos en } \\
\text { educación sobre la economía } \\
\text { circular. }\end{array}$ & $\begin{array}{l}\text { Vídeos didácticos de expertos abordando distintos tópicos sobre economía circular: } \\
\text { Tema 1. ¿Es adecuada la incorporación de los contenidos de desarrollo sostenible y economía } \\
\text { circular en el curriculo de primaria y secundaria? } \\
\text { Tema 2. ¿Qué medidas educativas se están desarrollando para concienciar y sensibilizar a los } \\
\text { jovenes en esta materia? } \\
\text { Tema 3. ¿Qué metodologías educativas son más efectivas para provocar una sensibilización } \\
\text { en los estudiantes? } \\
\text { Tema 4. ¿Cuáles son los recursos digitales más eficaces para trabajar en el aula este tipo de } \\
\text { temáticas y provocar una mayor sensibilización y concienciación? }\end{array}$ & On-Line & Marzo \\
\hline $\begin{array}{l}\text { Módulo 4. La voz de los estudiantes } \\
\text { sobre la economía circular. }\end{array}$ & $\begin{array}{l}\text { Elaboración de una Unidad didáctica y un video donde quede reflejado parte del conocimiento } \\
\text { del curso adquirido por los participantes y sus estudiantes. }\end{array}$ & On-line & Abril \\
\hline $\begin{array}{l}\text { Módulo 5. ¡Participa en el concurso } \\
\text { de videos didácticos sobre } \\
\text { economía circular. }\end{array}$ & $\begin{array}{l}\text { Concurso sobre el video realizado en el módulo } 4 \text {. Aunque no es obligatorio, los docentes, junto } \\
\text { a sus estudiantes podrán participar en el concurso subiendo a la web del proyecto el video } \\
\text { correspondiente. }\end{array}$ & On-line & Mayo \\
\hline
\end{tabular}

Figura 4. Módulos y contenidos del curso de formación docente.

\section{Acción 3: Concurso de videos acerca de la EC}

El principal factor para lograr el objetivo de sensibilización en torno a la EC es dar voz a los estudiantes a través de sus propios productos, lo cual ha sido contrastado por Gavilondo Rodríguez (2016) en cuanto al interés del estudiante en el video para visualizar mejor su trabajo.

Así, el concurso buscó que los estudiantes participaran, junto con sus profesores, en la creación de videos en torno a la EC, las mujeres en la ciencia y los elementos químicos. Para la selección, evaluación y gratificación consideramos la calidad de las gamificaciones, el formato y los contenidos trabajados (Gértrudix et al., 2017).
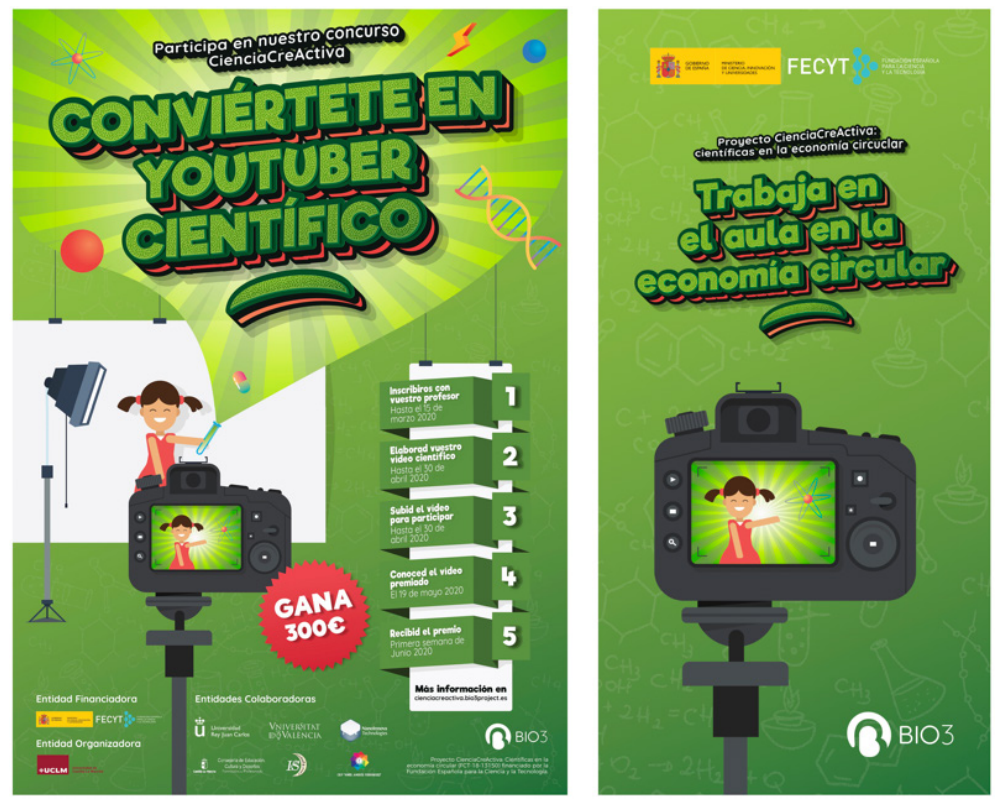

Figura 5. Póster y panfleto de promoción del concurso. 
Lamentablemente, debido a la pandemia del COVID $19-\mathrm{y}$ al estar los centros de educación en España con educación on line-, la entrega de los premios se realizó a través de videoconferencia.

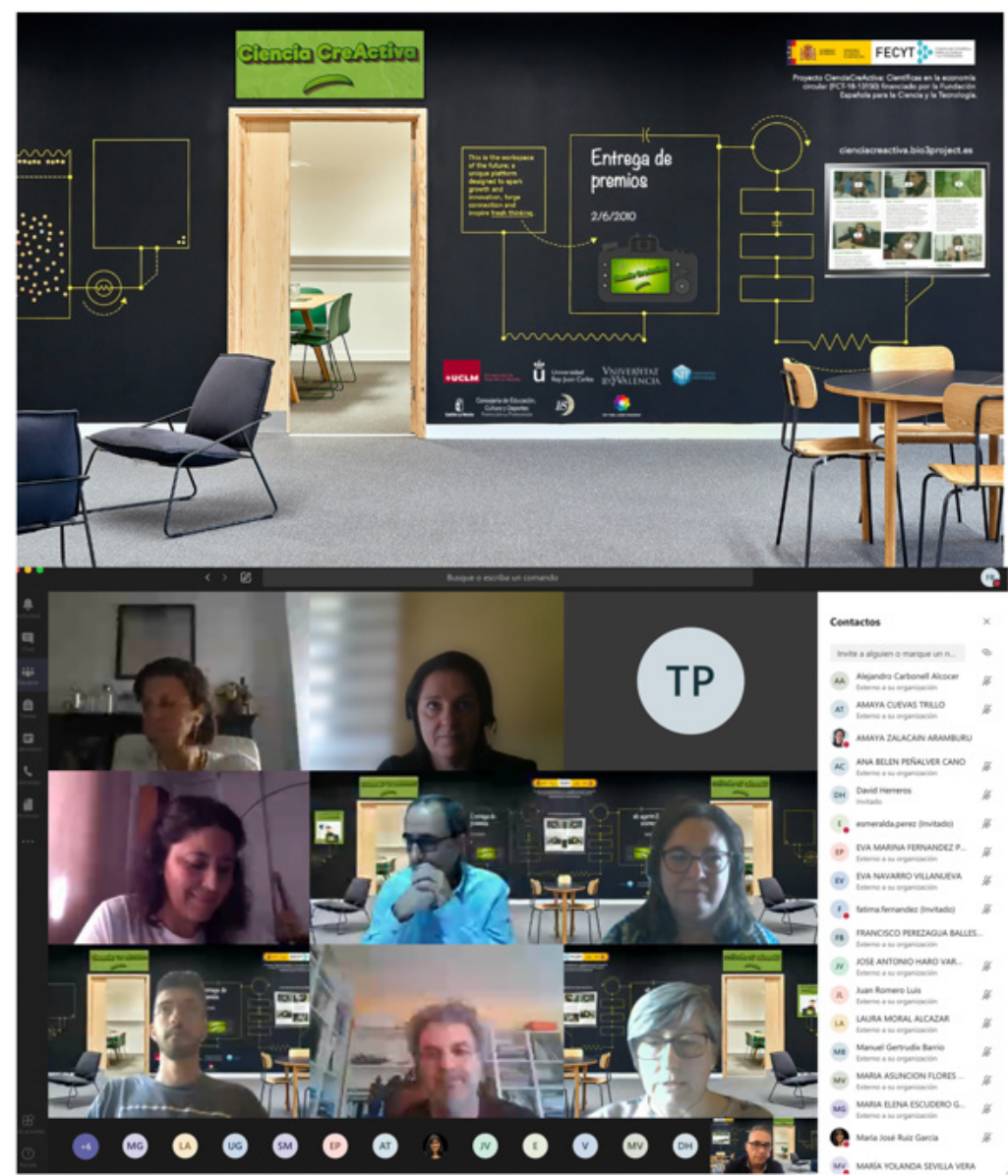

Figura 6. Entrega de premios virtualmente.

\section{Conclusiones, discusión, y perspectiva de futuro}

\section{Estrategias para la transición}

A lo largo del texto hemos podido evidenciar la necesidad de un cambio de paradigma en el sistema de producción y consumo. Para tener una base sólida en la cual cimentar un proyecto a largo plazo es necesario disponer de un marco internacional favorable, que permita y fomente el desarrollo de investigaciones que aboguen por este cambio (Comisión Europea, 2014; European Comission, 2017).

No obstante, son varios los autores que evidencian que las barreras culturales impiden que la EC pase de ser un discurso para la minoría a una idea generalizada (Canal Vieira \& Gonçalves Amaral, 2016; Kirchherr et al., 2018). Como expone Enrique Leff (2017), la ecología política debe construirse desde la diversidad "a través de una política de la diferencia y una ética de la otredad. Esta es la visión que abre el diálogo creativo entre las ecologías políticas 
regionales del mundo encontradas y atravesadas por sus estrategias de poder” (p. 251), esto es, una epistemología política en la que debe ser abordado un estudio profundo del "saber de las culturas sobre su organización social y productiva, sobre su relación con la naturaleza y con el orden económico dominante” (Leff, 1998, p. 233).

Vivimos en un mundo dominado por el neoliberalismo que responde más a un tipo de estrategia política que a los imperativos económicos subyacentes, por lo que debemos buscar una racionalidad ambiental en la que se dé primacía a la diversidad cultural, "se cuestione la sobreeconomización del mundo, el desbordamiento de la racionalidad cosificadora de la modernidad, de los excesos del pensamiento objetivo y utilitarista" (Leff, 2004, p. X del prólogo). Al respecto, en 1971 Eduardo Galeano en su ensayo Las venas abiertas de América Latina ya comentaba que "la división internacional del trabajo consiste en que unos países se especializan en ganar y otros en perder" (p. 15), situación que también critica la filósofa canadiense Naomi Klein (2000): “The Third World, as they say, has always existed for the comfort of The First" (p. 34), cuando argumenta que las zapatillas Nike, las muñecas Barbie o el café de Starbucks son fabricados en los talleres de Vietnam, Sumatra o Guatemala, merced al neoliberalismo y la globalización imperante. Así, lejos de encontrarnos en una situación de progreso, cada ańo que pasa nos situamos con un mundo más desigual, donde las brechas no son solo tecnológicas, sino que la otredad del norte sobre el sur se hace patente también en un colonialismo científico (Espinosa, 2020).

Por todo esto, implantar el modelo de EC conlleva muchos retos. Entre ellos está el de cambiar la mentalidad, los paradigmas impuestos y los hábitos del consumismo moderno, donde la preferencia por la exclusividad y la autenticidad minan los principios de la EC del reciclaje y la reutilización (Hood, 2016).

\section{La educación para el desarrollo sostenible}

El estudio del reciclaje en todos los niveles educativos ha resultado ser una experiencia enriquecedora. El aumento del interés por aprender y mejorar en la competencia natural se ha evidenciado en los últimos ańos, puesto que este tipo de educación hace intervenir la multidisciplinariedad entre campos como la ciencia, la química, la mecánica, la eléctrica, etc. No obstante, el beneficio va más allá del meramente educacional, ya que el trabajo en la importancia del reciclaje y los efectos del cambio climático trasciende hacia una necesidad de cambio de paradigma que puede permitir una didáctica renovada acerca de un modelo alternativo, como es la EC.

Llevamos casi 50 años de advertencias desatendidas con fundamentos científicos ante una "situación de emergencia de escala planetaria” (EsXrebellion, 2019, primer párrafo), y, aunque el poder económico está globalizado y no atiende a una verdadera democracia ambiental como ontología política donde puedan "vivir muchos mundos en este mundo" (Leff, 2017, p. 248), las pequeñas acciones educativas como las que hemos presentado pueden ayudar a los más jóvenes a entender esa racionalización ambiental a la que alude Enrique Leff (2004) y así discernir que ciertas acciones humanas perjudican no solo nuestro entorno más próximo, sino que repercuten negativamente en la forma de vida de otros pueblos y contextos.

Esta concienciación debe empezar en los primeros niveles, donde ya existen muestras acerca de cómo ayudar a trabajar el reciclaje en el aula, pues se crea con ello una conciencia en ciudadanos que, en su mayoría, no han tenido experiencias externas similares y que deberán lidiar con los efectos en un futuro a largo plazo.

\section{Recursos y materiales para una sensibilización y cambio hacia una EC}

Hemos podido comprobar que existen ciertos recursos y materiales didácticos que se ajustan más eficientemente a la hora de provocar una mejora educativa en el conocimiento y sensibilización en torno a la EC en estudiantes de preuniversitarios. En este sentido, Internet y las tecnologías avanzadas ayudan a potenciar la conexión entre el aprendiz y los contenidos, influyendo en el comportamiento humano y en cómo evaluar el cambio de conductas. 
El diseño persuasivo, —que se basa en influenciar el comportamiento humano a través de las características de un material—, es capaz de potenciar ciertos objetivos del desarrollo sostenible como, por ejemplo, el control del malgasto de comida como forma de regenerar el desarrollo económico y ambiental (Fogg, 2009).

Otro aspecto es la gamificación, que basa su idea en que disfrutar un juego es una manera efectiva de aprender (Singer, Golinkoff \& Hirsh-Pasek, 2006). Este recurso educativo permite limar las diferencias y conseguir que el objetivo sea más real y cercano en la implementación de la educación ambiental, por ejemplo con la realidad virtual, ya que existen evidencias de cómo provocan cambios en el comportamiento de los estudiantes (McComas, 2002).

\section{La educación como valor de futuro}

Ante este encorsetamiento en el que se halla la transición hacia la EC, la escuela puede tener la llave para un futuro sostenible en el que el modelo de consumo sea más respetuoso con el medio ambiente y más comprometido con el planeta (Buckland, 2007). Lo hemos podido comprobar en el proyecto Ciencia CreActiva, donde el valor de los productos creados por los estudiantes y dirigidos por sus profesores han producido una conciencia y sensibilización positiva ante los elementos estudiados de la EC. El trabajo de este tipo de realidades en la educación puede ir formando ciudadanos críticos con una conciencia ecológica que permita avanzar en el futuro.

Por otro lado, el docente debe ser el mejor guía para ayudar a cambiar y sensibilizar a sus estudiantes creando el deseo de aprendizaje (Jay, 2014), lo cual implica para este obtener una formación específica en temáticas actuales que le ayudarán a continuar con su labor docente y, por ende, contribuirán, de forma clara, a guiar a sus estudiantes en temáticas como la EC (los estudiantes esperan que el docente le entregue cosas enseñar- que no pueden encontrar en Internet).

Además, el trabajo y las acciones educativas en torno a un elemento concreto como el que se ha trabajado en este artículo, fomentan la convivencia (Valencia Torres, González Suárez y Valencia Torres, 2017) entre los participantes de la actividad pedagógica, ya que viven unos con otros sobre la base de una serie de relaciones sociales y códigos (Rodríguez y Vaca, 2010), aprendiendo de sus propias experiencias y de las de los demás, promoviendo con ello el desarrollo crítico, el cuidado y la corresponsabilidad (Vilches, Gil Pérez, Toscano y Macías, 2014).

En el proyecto hemos podido dar cuenta de cómo una intervención integral en el aula ayuda a una mejor sensibilización y conocimiento en torno a los elementos de la EC. Aun así, todavía no conocemos los efectos que esta podrá tener a medio y largo plazo.

De igual modo, sabemos que existen ciertos factores que dificultan una integración normalizada de contenidos como los que hemos presentado aquí, entre los que se cuenta la existencia de un currículo cerrado, la escasa formación del profesorado, la casi nula interdisciplinariedad y, por lo tanto, de un modelo de temporalización horaria y atomización de los contenidos. Con todo, debemos pasar a una acción crítica y sostenible en el tiempo donde los estudiantes comprendan el problema como una oportunidad de futuro, analizando todas las perspectivas, ya que muchas veces - como ocurre con las enfermedades - la gente tiene tanto miedo a la propia enfermedad como a la posible vacuna que la elimine: el ecoagobio versus "no pasa nada" (Sáez, 2019).

Esta situación crea la necesidad de plantear un proyecto interdisciplinar, pues no existe la flexibilidad necesaria para trabajarlo de manera holística, como sí lo permite, por ejemplo, el sistema de la educación infantil, que puede ser objeto de estudio por su versatilidad y capacidad del alumnado de concienciación y sensibilización (Brenes Gómez, 2016). Por ello, una continuidad en el proceso con nuevos retos, y haciendo partícipe a un mayor número de docentes y estudiantes permitiría garantizar una consolidación del proyecto y, sobre todo, desde un trabajo didáctico integrador, interdisciplinar, internivelar y transversal, puesto que se trata de un tema que importa a todos, por lo que todos debiéramos ser partícipes del mismo. 
"Convertid un árbol en leña y arderá para vosotros, pero no producirá flores ni frutos para vuestros hijos" (Rabindranath Tagore)

El artículo original fue recibido el 2 de junio de 2020

El artículo revisado fue recibido el 16 de agosto de 2020

El artículo fue aceptado el 31 de agosto de 2020

\section{Referencias}

Aguilar Luzón, M. C., García Martínez, M. A., Monteoliva Sánchez, A., y Salinas Martínez de Lecea, J. M. (2006). El modelo del valor, las normas y las creencias hacia el medio ambiente en la predicción de la conducta ecológica. Revista Internacional de Psicología Ambiental, 7(2), 21-44. Recuperado de https://cutt.ly/iyZBGNB

Åhlberg, M. K., Aineslahti, M., Alppi, A., Houtsonen, L., \& Nuutinen, A. M. (2014). Education for sustainable development in Finland. En R. Jucker \& R. Mathar (Eds.), Schooling for sustainable development in Europe (221-239). Springer Link: Luxemburg.

Anderberg, M. \& Thisted, S. (2015). Swedish waste streams in a circular economy-a study of the perception change of waste flows when reviewing waste recycling target for the EU proposed circular economy package (Master thesis). Lund University, Lund.

Apud Porras, A. (Ed.). (2012). El consumo responsable en centros educativos. Red Valenciana de Educadores y Educadoras para la Ciudadania Global. Recuperado de https://cutt.ly/wyXrAOS

Avilés Pozo, A. (31 de julio de 2018). Castilla-La Mancha inicia la batalla legislativa contra el desperdicio alimentario. El Diario.es (s/p). Recuperado de https://goo.gl/Ss9Sz1

Barth, J., Kim, H., Eno, C., \& Guadagno, R. (2018). Matching abilities to careers for others and self: Do gender stereotypes matter to students in advanced math and science classes? Sex Roles, 79(1-2), 83-97. https://doi.org/10.1007/s11199-017-0857-5

Bio3 (2020). Ciencia CreActiva. Recuperado de https://cienciacreactiva.bio3project.es

Boudes, Ph. (2011). United Nations Conference on the Human Environment. En J. Newman (Ed.), Green ethics and philosophy-the green series: Toward a sustainable environment, Vol. VIII (pp. 410-413). London: Sage publications.

Brenes Gómez, E. (2016). Educar para el consumo responsable en la etapa de infantil. Una propuesta metodológica desde la sostenibilidad. (TFG). Recuperado de https://goo.gl/yDVHzn

Buckland, H. (Ed.) (2007). "Piénsatelo bien". Reflexiones sobre el papel de la educación para un consumo más responsable. Barcelona: El Tinter. Recuperado de https://goo.gl/DiuYX3

Canal Vieira, L. \& Gonçalves Amaral, F. (2016). Barriers and strategies applying cleaner production: A systematic review. Journal of Cleaner Production, 113(1), 5-16. https://doi.org/10.1016/j.jclepro.2015.11.034

Centro para el Desarrollo Tecnológico Industrial, CDTI. (2016). Horizonte 2020: Novedades y lecciones aprendidas. Recuperado de https://cutt.ly/5gvUXZi

Chen, C. W. K. \& Chen, Y. T. (2019). Informal education of aerosol science by animation. Theory and practice. IOP Conf. Series: Earth and Environmental Science 373 012002. http://dx.doi.org/10.1088/1755-1315/373/1/012002

Comisión Europea, CE. (2013). Un marco para las políticas de clima y energía en 2030. Recuperado de https://cutt.ly/WyLxwZM

Comisión Europea, CE. (2014). Estrategias nacionales y regionales para la especialización inteligente (RIS3). Recuperado de https://goo.gl/v19hCW

Dunlap, R., Van Liere, K., Mertig, A., \& Jones, R. (2000). New Trends in Measuring Environmental Attitudes: Measuring endorsement of the new ecological paradigm: A revised NEP Scale. Journal of Social Issues, 56(3), 425-442. https://doi.org/10.1111/0022-4537.00176 
Ecoembes.es (s/f) ¿Y si hacemos algo más? Recuperado de https://goo.gl/nY9ha8

Economía Circular (2017). Por qué y cómo desarrollar estrategias de economía circular en el ámbito regional. Recuperado de https://goo.gl/YcPKnK

España Circular 2030 (2018). Estrategia española de economía circular. Ministerio de Agricultura y Pesca, Alimentación y Medio Ambiente, MAPAM y Ministerio de Economía, Industria y Competitividad, MEIC. Recuperado de https://goo.gl/3SFDP4

Espinosa, C. I. (12 de agosto de 2020). El colonialismo científico, una realidad tenaz. El Diario.es. Recuperado de https://cutt.ly/Vd8ZRDD

EsXrebellion (2019). 50 años de advertencias desatendidas. Recuperado de https://telegra.ph/50-años-de-advertencias-desatendidas-05-21

European Comission (2017). Results of Horizon 2020 stakeholder consultation: Interim evaluation of Horizon 2020. Recuperado de https://goo.gl/37apcA

Fernández, J. A. (16 de julio de 2018). ¿¿Sabes qué es la economía circular? Estos seis niños lo tienen claro. El País (Proyecto Naturgy). Recuperado de https://goo.gl/wvU9Lg

Fernández-Cézar, R., Pinto-Solano, N., y Muñiz-Hernández, M. (2018). ¿Mejoran los proyectos de divulgación con experimentación la actitud hacia las clases de ciencias? Revista de Educación, 381, 285-308. Recuperado de https://dialnet.unirioja.es/servlet/articulo?codigo $=6518464$ \&orden=0\&info=link

Fogg, B. J. (2009). A behavior model for persuasive design. Persuasive '09: Proceedings of the 4th International Conference on Persuasive Technology. https://doi.org/10.1145/1541948.1541999

Fundación COTEC para la Innovación. (2019). Situación y evolución de la economía circular en España. Informe 2019. Recuperado de https://cutt.ly/ByZhE1G

Galeano, E. (1971). Las venas abiertas de América Latina. México, D.F.: Siglo XXI editores.

Gavilondo Rodríguez, C. (2016). La producción de videos científicos: un acercamiento teórico. Alteridad, 11(1), 254-264. Recuperado de https://www.learntechlib.org/p/195393/

Gértrudix, M., Rajas, M., Barrera, D., Bastida, M., y Soto, C. (2017). Realización de vídeo educativo: análisis de la producción audiovisual de los MOOC de URJCx. Madrid: McGraw Hill Education.

Gobierno de Espańa y Comisión Europea, CE. (2017). Pacto por una economía circular: el compromiso de los agentes económicos y sociales 2018-2020. Recuperado de https://goo.gl/c7vEcj

Gudynas, E. (2015). Derechos de la naturaleza. Buenos Aires: Tinta Limón.

Gudynas, E. (2018). Extractivismos: el concepto, sus expresiones y sus múltiples violencias. Papeles de Relaciones Ecosociales y Cambio Global, 143, 61-70. Recuperado de https://cutt.ly/qd8B2GY

Guo, P. \& Zhang, X. (2014). Study on circular economy education in rural areas in China. Proceedings of the International Conference on Logistics, Engineering, Management and Computer Science (pp. 24-26). Paris: Atlantis Press. https://dx.doi.org/10.2991/lemcs-14.2014.213

Hood, B. (2016). Make recycled goods covetable. Nature, 531(special issue), 438-440. Recuperado de https://cutt.ly/fyXe83W

Inocêncio da Silva, R. P. \& Ferreira Maia, F. J. (2017). A aplicaçâo da extrafiscalidade à Política Nacional de Resíduos Sólidos examinada pela perspectiva de Enrique Leff. Revista Acadêmica Faculdade de Direito do Recife, 89(1), 50-71. Recuperado de https://cutt.ly/1d8Koep

Jay, M. (13 de marzo de 2014). Un docente debe crear deseo de aprendizaje. Tiching blog. Recuperado de http://bit.ly/Na1bdn

Kaplan, J. (2015). Humans need not apply: A guide to wealth and work in the age of artificial intelligence. New Haven: Yale University Press.

Klein, N. (2000). No logo. Toronto: Alfred A. Knopf.

Kirchherr, J., Piscicelli, L., Bour, R., Kostense-Smit, E., Muller, J., Huibrechtse-Truijens, A., \& Hekkert, M. (2018). Barriers to the circular economy: Evidence from the European Union (EU). Ecological Economics, 150, 264272. https://doi.org/10.1016/j.ecolecon.2018.04.028

Leff, E. (s.f.). Globalización, racionalidad ambiental y desarrollo sustentable. Recuperado de https://cutt.ly/wd8Zxfa

Leff, E. (1998). Saber ambiental, sustentabilidad, racionalidad, complejidad, poder. México, D.F.: Siglo XXI editores.

Leff, E. (2004). La racionalidad ambiental. México, D.F.: Siglo XXI editores. 
Leff, E. (2017). Las relaciones de poder del conocimiento en el campo de la ecología política. Ambiente \& Sociedade, 20(2), 229-262. Recuperado de https://cutt.ly/Gd9PoSM

Ley 22/2011, de 28 de julio, de Residuos y Suelos Contaminados. BOE núm. 181, de 29/07/2011. 1-52. Recuperado de https://cutt.ly/nyZgu7Y

Ley 7/2019, de 29 de noviembre, de Economía Circular de Castilla-La Mancha. [2019/11104]. Diario Oficial de Castilla-La Mancha, 12 de diciembre de 2019, 48437-48442. Recuperado de https://cutt.ly/JyZhUX2

Macedo, B. y Salgado, C. (2007). Educación ambiental y educación para el desarrollo sostenible en América Latina. Revista Fórum de Sostenibilidad, 1, 29-37. Recuperado de https://cutt.ly/Ggvv8JA

Maya, A. (1998). El retorno a la tierra: introducción a un método de interpretación ambiental. Bogotá: Ministerio de Educación-Ministerio del Medio Ambiente-Universidad Nacional de Colombia, Instituto de Estudios Ambientales.

McComas, W. F. (Ed.). (2002). The nature of science in science education. Rationales and strategies. Netherlands: Springer Nature.

Ministerio de Medio Ambiente (2010). Encuesta de ecología y medio ambiente. Centro de Investigaciones Científicas (CSIC). Recuperado de http://www.cis.es/cis/opencm/EN/2_bancodatos/estudios/ver.jsp?estudio=11404

Molano, A. B. (2012). La complejidad de la educación ambiental: una mirada desde los siete saberes necesarios para la educación del futuro de Morin. Revista de Didáctica Ambiental, 8(11), 1-9. Recuperado de https://cutt.ly/Kgvbris

Monclus, A. y Saban, C. (1999). Educación para la paz. Madrid: Síntesis.

Organizaciones de las Naciones Unidas, ONU. (1973). Informe de la Conferencia de las Naciones Unidas sobre el Medio Humano. Estocolmo, 5 a 16 de junio de 1972. Recuperado de https://cutt.ly/gdEBULF

Pearce, D. W. \& Turner, R. K. (1990). Economics of natural resources and the environment. New York: Harvester Wheatsheaf.

Ramírez, A., Sánchez, J. M., y García, A. (2004). El desarrollo sustentable: interpretación y análisis. Revista del Centro de Investigación. Universidad La Salle, 6(21), 55-59. Recuperado de https://www.redalyc.org/articulo.oa?id=34202107

Rodríguez, M. C. y Vaca, P. (2010). Promover la convivencia escolar: una propuesta de intervención comunitaria. Aletheia, 33, 179-189. Recuperado de https://www.redalyc.org/pdf/1150/115021494016.pdf

Ruiz Medina, D., Parga Lozano, D., y Martínez Pérez, L. (2009). Creencias de los profesores de preescolar y primaria sobre ciencia, tecnología y sociedad, en el contexto de una institución rural. TED, 25, 41-61. https://doi.org/10.17227/ted.num25-467

Ruiz-Pastor, L., Mulet, E., y Chulvi, V. (2018). Análisis de la oferta educativa sobre economía circular en la educación superior española. Recuperado de https://cutt.ly/XyZOYWL

Salvia, R., Andreopoulou, Z. S., \& Quaranta, G. (2018). The circular economy: A broader perspective for rural areas. Rivista di studi sulla sostenibilità, 1, 87-105. http://dx.doi.org/10.3280/RISS2018-001008

Sánchez-Emeterio, G. \& Figueira, C. (2019). Eco-social literacy: Circular economy conceptions in initial teacher training. Proceedings of the 9th International Conference the Future of Education, 486-490.

Sandoval-Ruiz, C. E. y Ruiz-Díaz, E. (2019). Eco-innovación en ingeniería de alimentos sostenible aplicando técnicas inteligentes de eficiencia energética-ecosveg. Universidad, Ciencia y Tecnología, 22(87), 54-66. Recuperado de https://cutt.ly/xyZhxwP

Santiago, R. (18 de mayo de 2015). ¿Qué es micro-learning? The flipped classroom. Recuperado de https://cutt.ly/KyXcDEv Sáez, S. (2 de abril de 2019). Eco-ansiedad: ¿quién teme al fin del mundo? Lamarea.com. Recuperado de https://cutt.ly/Gd8VGeI Seguí, L., Medina, R., y Guerrero, H. (2018). Gestión de residuos y economía circular. Recuperado de https://cutt.ly/vyZgvJq

Singer, D. G., Golinkoff, R. M., \& Hirsh-Pasek, K. (Eds.). (2006). Play = learning: How play motivates and enhances children's cognitive and social-emotional growth. Oxford: Oxford University Press. https://doi.org/10.1093/acprof:oso/9780195304381.001.0001

Soto, M. (2018). Humanidad necesita 1,7 planetas para satisfacer su ritmo de consumo. Recuperado de https://cutt.ly/UyZgmKk 
Stadler, M. M., Baganz, D., Vermeulen, T., \& Keesman, K. J. (2015). Circular economy and economic viability of aquaponic systems: Comparing urban, rural and peri-urban scenarios under Dutch conditions. Proceedings of ICESC2015: Hydroponics and Aquaponics at the Gold Coast, 1176, 101-114.

https://doi.org/10.17660/ActaHortic.2017.1176.14

Stahel, W. \& Reday, G. (1981). Jobs for tomorrow, the potential for substituting manpower for energy. New York: Vantage Press.

Tisserant, A., Pauliuk, S., Merciai, S., Schmidt, J., Fry, J., Wood, R., \& Tukker, A. (2017). Solid waste and the circular economy: A global analysis of waste treatment and waste footprints. Journal of Industrial Ecology, 21(3), 628-640. https://doi.org/10.1111/jiec.12562

Torres Consuegra, E. (1996). ¿Cómo lograr la educación ambiental en tus alumnos? La Habana: Ed. Pueblo y Educación.

Valencia Torres, M. A., González Suárez, E., y Valencia Torres, G. A. (2017). Érase una vez una escuela que quería aprender a convivir... la versión de los profesores. San Luis de Potosí: Congreso Nacional de Investigación Educativa.

Vilches, A., Gil Pérez, D., Toscano, J. C., y Macías, O. (2014). Educación para la Sostenibilidad. Un compromiso renovado para la educación para la sostenibilidad. Recuperado de http://www.oei.es/decada/accion.php?accion=2

Vozmediano Sanz, L. y San Juan Guillén, C. (2005). Escala nuevo paradigma ecológico: propiedades psicométricas con una muestra española obtenida a través de Internet. Medio Ambiente y Comportamiento Humano, 6(1), 39-47. Recuperado de https://cutt.ly/kyZBUjg

Wadel G., Pérez, L., Giacomelli, F., Scheffer, A., y Pascual; G. (2018). PRECO: Red Universitaria de Aprendizaje sobre Aplicación de Reciclados. X Congreso Regional de Tecnología en Arquitectura (CRETA) (La Plata, 2018), 284-293. Recuperado de https://cutt.ly/myZhRXV

Webster, K. (2017). The circular economy: A wealth of flows. Ellen MacArthur Foundation Publishing.

Whalen, K. A., Berlin, C., Ekberg, J., Barletta, I., \& Hammersberg, P. (2017). 'All they do is win': Lessons learned from use of a serious game for circular economy education. Resources, Conservation and Recycling, 135, 335-345. https://doi.org/10.1016/j.resconrec.2017.06.021

Whitmore-Williams, S. Cl., Manning, Ch., Krygsman, K., \& Speiser, M. (2017). Mental health and our changing climate: Impacts, implications, and guidance. American Psychological Association (APA). Recuperado de https:// cutt.ly/Sd8C958 